\title{
Ascending Aorta and Total Aortic Arch Replacement in a Child with Infective Endarteritis
}

\author{
Ting Lu ${ }^{1}$, Yifeng Yang ${ }^{1}$ and Can Huang ${ }^{1, *}$ \\ ${ }^{1}$ The Second Xiangya Hospital of Central South University, Changsha, China \\ "Corresponding author: The Second Xiangya Hospital of Central South University, Changsha, China. Email: huangcan413@csu.edu.cn
}

Received 2018 September 24; Revised 2018 December 23; Accepted 2019 January 03.

Keywords: Ascending Aortic Stenosis, Ascending Aorta and Total Aortic Arch Replacement, Infective Endarteritis

Infective endarteritis, a special type of infective endocarditis caused by aortic stenosis, remains uncommon, although patent ductus arteriosus and aortic coarctation accounting for infective endarteritis have been reported in a few cases (1). Here, we report a 12-year-old boy with ascending aortic stenosis, who developed infective endarteritis and aneurysm, underwent a successful ascending aorta and total aortic arch replacement, which is extensively employed to treat aortic diseases.

A 12-year-old boy was admitted to our hospital on August 4, 2017 because of complaints of on and off fever for 1 month and intermittent claudication for 3 days. During admission, his body temperature was $36.7^{\circ} \mathrm{C}$ and heart rate was $112 \mathrm{bpm}$. Upon heart auscultation, grade 3 murmur could be heard at the $3 \sim 4$ intercostal space to the left of the sternum. Routine blood examination revealed white blood cells (WBC): $13.98 \times 109$ with a ratio of $91.1 \%$; erythrocyte sedimentation rate (ESR): $80 \mathrm{~mm} / \mathrm{h}, \mathrm{C}$ reactive protein (CRP): $91.7 \mathrm{~g}$; procalcitonin (PCT): $9.37 \mathrm{ng} / \mathrm{mL}$, antistreptococcal antibody (ASO): 144. Hemolytic Streptococcus sensitive to vancomycin was cultured from the blood. Cardiac color Doppler ultrasound examination indicated a possibility of ascending aortic stenosis, infective endocarditis, and reduced function of the heart. Computed tomography examination showed ascending aortic stenosis and infective aneurysm involved in the ascending aorta, aortic arch, brachiocephalic trunk, left common carotid artery, and left subclavian artery (Figure 1).

Intraoperative femoral artery and superior inferior vena cava intubation was performed to establish a cardiopulmonary bypass, while maintaining a body temperature of $28^{\circ} \mathrm{C}$. After blocking the aorta, the non-coronal sinus was split and enlarged by a Gore-Tex vessel to build a new aortic root. Subsequently, a 20-mm Gore-Tex artificial blood vessel was used to replace the ascending aorta, and an 18-mm Gore-Tex three-branch vessel was employed to instead of the active artery arch.

During the operation, the aortic wall tissue was sent to the pathological examination. It revealed the formation of a fibrous thrombus-like substance and infiltration of numerous lymphocytes, plasma cells, and granulocyte in the aortic wall. During the perioperative period, the patient was administered vancomycin for 6 weeks. Cardiac color Doppler ultrasound and CT examination of anastomotic stoma were regularly performed to evaluate the recoverment, which showed that the patient's cardiac function was improved significantly.

The incidence of infective endocarditis due to a congenital heart defect is $6 \%-24 \%$ (2). Infective endocarditis often occurs in the aortic valve, but it rarely affects the ascending aorta and the aortic arch. Infective endarteritis is typically seen in patients with aortic coarctation and silent patent ductus arteriosus. Staphylococcus aureus and Streptococcus viridans are the most common infecting organisms (1). This patient was diagnosed with severe ascending aortic stenosis in our hospital two years ago and had no lesion in the aortic valve. In this hospitalization, alpha hemolytic Streptococci were cultured from the blood and the pathological examinations of the vascular wall tissues showed a large number of neutrophil infiltrations. Therefore, we considered this a case of aortic inflammation caused by ascending aortic stenosis. As we know, this is the first patient of infective endarteritis caused by the isolated ascending aortic stenosis.

Ascending aorta and aorta arch replacement is widely used in adult aortic dissections. In children, it is mainly used for those with ascending aorta dilation caused by connective tissue disease or Ross surgery (3). However, due to the lack of growth in the tissues after replacement, its use in children is limited. In 2003, Carrel (3) reported that 

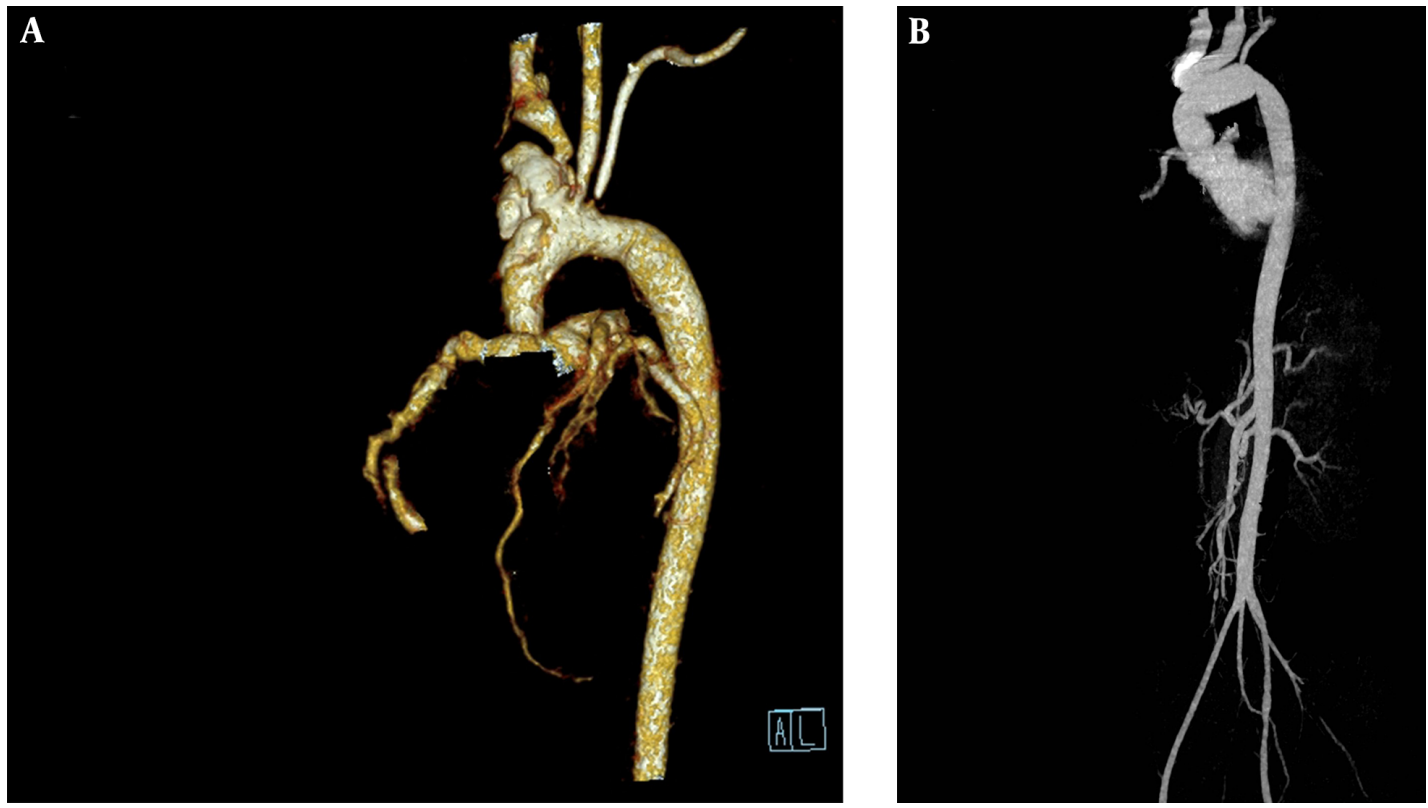

Figure 1. Results of preoperative (A) and postoperative (B) multidetector computed tomography examination

26 cases of aortic root dilatation were treated with aortic root repair, and their short-term effects were better. However, serious complications were the main cause of early death. In 2012, a 2.5-year-old child with Loeys-Dietz syndrome (LDS) received ascending aorta and aortic arch replacement treatment and was reported to be better than prosthetic valve replacements (4). In the present case, we used a two-vessel blood vessel and a 10-mm Gore-Tex blood vessel to construct a three-vessel blood vessel in place of the aortic arch because of the lack of a matching threevessel blood vessel. For the non-growth of Gore-Tex blood vessels, there may still be a possibility of reoperation in a later period, and further observation and follow-up are needed.

This patient had been diagnosed with ascending aortic stenosis two years earlier. However, the family members had given up surgical treatment because of economic problems and patient's high surgical risk. Although the infective endocarditis rarely affects the ascending aorta and the aortic arch, surgical treatment should be performed as early as possible in children with ascending aortic stenosis to avoid even more serious complications (1). At present, the Chinese government provides better financial support for the treatment of congenital heart diseases, which will further improve the curative rate of children with congenital heart disease in China.

\section{Footnotes}

Conflict of Interests: We declare that we have no conflict of interest.

Ethical Considerations: All procedures performed in studies involving human participants were in accordance with the ethical standards of the Institutional and/or National Research Committee and with the 1964 Helsinki Declaration and its later amendments or comparable ethical standards.

Funding/Support: This study was supported by Hunan Provincial Nature Science Foundation of China (14JJ3037).

\section{References}

1. Jaleleddine Z, Sana C, Faker G, Adel K. Infective endarteritis and false mycotic aneurysm complicating aortic coarctation. Ann Pediatr Cardiol. 2012;5(2):197-9. doi: 10.4103/0974-2069.99627. [PubMed: 23129914]. [PubMed Central: PMC3487213].

2. Franco-Paredes C, Workowski K, Harris M. Infective endocarditisendarteritis complicating coarctation of the aorta. Am J Med. 2002;112(7):590-2. [PubMed: 12015258].

3. Carrel T, Berdat P, Pavlovic M, Sukhanov S, Englberger L, Pfammatter JP. Surgery of the dilated aortic root and ascending aorta in pediatric patients: Techniques and results. Eur J Cardiothorac Surg. 2003;24(2):249-54. doi: 10.1016/s1010-7940(03)00302-6. [PubMed: 12895616].

4. Ozker E, Vuran C, Saritas B, Turkoz R. Valve-sparing replacement of the ascending aorta and aortic arch in a child with Loeys-Dietz syndrome. Eur J Cardiothorac Surg. 2012;41(5):1184-5. doi: 10.1093/ejcts/ezr235. [PubMed: 22228852]. 